\title{
Computational study of chemical reactions during heat and mass transfer in in magnetized partially ionized nano-liquid
}

\author{
M. Nawaz ${ }^{a 1}$ and Shafia Rana ${ }^{a}$ \\ ${ }^{a}$ Department of Applied Mathematics \& Statistics, Institute of Space Technology, \\ Islamabad, 44000, Pakistan
}

\begin{abstract}
Homogeneous and heterogeneous chemical reactions in partially ionized magnetonano-liquid are investigated theoretically using finite element method (FEM). The effects of ions and electrons collisions on the transport of heat and mass are analyzed for both the cases of heterogeneous and homogeneous chemical reactions. The simultaneous effects of dispersion of nanosized particles in partially ionized nano-liquid in the presence of magnetic field are also investigated. Through numerical experiments, it is noted that the temperature of partially ionized nano-liquid increases when electrons collision rate and ion collisions are increased. The transport rate of reacting species decreases when heterogeneous and homogeneous chemical reactions strengths are increased. It is also observed that the effect of electron collisions on the flow in $y$-direction is the same to that of ion collisions on the flow in $y$-direction. Homogeneous and heterogeneous chemical reactions have similar effects on concentration of chemically reacting species in qualitative sense. However, in quantitative sense, homogeneous chemical reaction has more significant effect on the concentration reacting species as compared to heterogeneous chemical reaction.
\end{abstract}

Keywords: Homogeneous and heterogeneous reactions, Hall and ion-slip currents, nanofluid, MHD, partially ionized.

\section{Introduction}

Theoretical study of physical happening through mathematical modeling using governing laws is of a great importance as this way of studying is the cheapest way of analyzing the under lying physics. Alternatively experimental study requires equipment set up which costs in term of money and time. Contrary to this, theoretical investigations require less resources and provide more accurate scientific information. Like other theoretical sciences, theoretical and computational chemistry is the most famous field of the chemistry. Recently, a much attention is given to computational chemistry and homogeneous and heterogeneous chemical

\footnotetext{
${ }^{1}$ Coresponding author: nawaz_d2006@@yahoo.com (Dr. Muhammad Nawaz)
} 
reactions in liquid regime are investigated theoretically. For example, Merkin [1] proposed a mathematical model for homogeneous-heterogeneous chemical reactions and investigated Blasius flow in the presence of heterogeneous-homogeneous reaction in Newtonian fluid over an isothermal surface. Hayat et al. [2] analyzed the effects of heterogeneous and homogeneous chemical reactions on the diffusion of chemically reacting species in Newtonian nano-fluid exposed to applied magnetic field. However, they considered neutral fluid, that is not composed of charge particles and electrons. Bachok et al. [3], theoretically, studied simultaneous effects of heterogeneous-homogeneous chemical reactions on the diffusion phenomenon of chemically reacting species in fluid in fluid regime over a surface moving with non-uniform velocity. It is noted that this work does not consider partially ionized liquid. Greesha et al. [4] investigated nonlinear convection in the presence of homogeneous-heterogeneous chemical reactions in a dual non-Newtonian fluid (Casson-Carreau) using Runge-Kutta numerical method. Hayat et al. [5] discussed simultaneous effects of heterogeneous chemical reactions and dispersion of nano-particles in a neutral fluid over a rotating disk. Again it is noted that they have not considered partially ionized liquid. Sajid et al. [6] investigated homogeneous-heterogeneous chemical reactions in MHD neutral fluid containing $\mathrm{Fe}_{3} \mathrm{O}_{4}$ nano-particles. Qayyum et al. [7] analyzed homogeneous -heterogeneous chemical reactions in reacting species in a neutral fluid emitting thermal radiations in a nonlinear manner.

The partially ionized liquid in the presence of applied magnetic field experiences forces of more than one kinds. These forces include magnetic force due to applied magnetic field, force due to the collision of electrons called Hall force, force due to the collision of ions called ion slip force. It is experimentally verified that Hall and ion slip forces are opposite to the force due to the applied magnetic field. Modelling of flow of partially ionized liquid can be done using physical laws including conservation of mass [8], momentum [8], energy equation [8], set of Maxwell equations [8]. The Forces due to Hall and ion slip currents can be calculated by using generalized Ohm's law [9]. Several studies on magnetohydrodynamic flow of partially ionized liquid using laws mentioned in [8] have been discussed. Here, latest investigations are described. For instance, the studies mentioned in references [10-21] and, the references there in, discussed various effects on partially ionized liquid but they do considered homogeneous-heterogeneous chemical reactions. The studies $[13,17]$ consider discuss the effect of dispersion of nano-particles on the enhancement of heat transfer without considering transport of chemically reacting species. Obviously, they have not considered homogeneous- 
heterogeneous chemical reactions in partially ionized liquid.

Now, it is verified fact that inclusion of nanosized particles in the pure fluid changes thermal properties (viscosity, thermal conductivity, electrical conductivity). Different empirical and theoretical models for effective viscosity, effective thermal conductivity and electrical conductivity have been proposed. In this study, the models $[17,21]$ for effective thermal properties are investigate MHD partially ionized liquid in a chemically reacting regime subject to heterogeneous and homogeneous chemical reactions.

Literature review reveals that only the studies $[13,17]$ discuss magnetohydrodynamic flow of partially ionized liquid containing nanosized metallic particles. However, these studies $[13,17]$ do not consider homogeneous-heterogeneous chemical reaction of species in partially ionized liquid. Also, to the best of our knowledge, in the flow of partially ionized liquid dispersion of nano-particles, heterogeneous-homogeneous reactions, heat generation, Joule heating, thermal radiations are not considered simultaneously. This article considers above mentioned effects, simultaneously, on the transport of chemically reacting species in the presence of homogeneous-heterogeneous chemical reactions in partially ionized nano-liquid. These simultaneous consideration gives set of highly nonlinear coupled problems which are difficult to solve analytically but we are thankful to finite element method which make it possible. Finite element method (FEM) is a powerful technique which is successfully employed to nonlinear problems $[13,17,21-23]$. The rest part of this manuscript is organized in five sections. Mathematical modeling and normalization procedures are given in section two. Solution methodology is briefly discussed in section three. Section four is designated for results and their discussion. The last section contains the key observations of this study.

\section{Mathematical formulation}

Let us examined Hall and ion slip effects on three dimensional radiative flow of magnetized nanofluid. Fluid is over a nonlinear stretching sheet moving with velocity $\mathbf{V}_{w}=a(x+$ $y)^{n} \hat{\imath}+d(x+y)^{n} \hat{\jmath}$, (where $n$ is the power-law index) in the presence of non-uniform magnetic field $B_{\circ}(x+y)^{\frac{n-1}{2}}$. Nonlinear source/sink are also considered. Transportation of chemical species in the presence of homogeneous and heterogeneous reactions is taken into account. Homogeneous reactions for autocatalysis are 


$$
\begin{gathered}
A+2 B \rightarrow 3 B, \quad \text { rate }=k_{c} a b^{2} \\
A \rightarrow B, \quad \text { rate }=k_{e} a
\end{gathered}
$$

Nonlinear conservation laws under boundary layer approximations are given by

$$
\begin{gathered}
\frac{\partial u}{\partial x}+\frac{\partial v}{\partial y}+\frac{\partial w}{\partial z}=0 \\
\rho_{n f}\left(u \frac{\partial u}{\partial x}+v \frac{\partial u}{\partial x}+w \frac{\partial u}{\partial z}\right)=\mu_{n f} \frac{\partial^{2} u}{\partial z^{2}}-\frac{\sigma_{n f} B_{0}^{2}(x+y)^{n-1}}{\left[\left(1+\beta_{e} \beta_{i}\right)^{2}+\beta_{e}^{2}\right]}\left[\left(1+\beta_{e} \beta_{i}\right) u-\beta_{e} v\right], \\
\rho_{n f}\left(u \frac{\partial v}{\partial x}+v \frac{\partial v}{\partial x}+w \frac{\partial v}{\partial z}\right)=\mu_{n f} \frac{\partial^{2} v}{\partial z^{2}}-\frac{\sigma_{n f} B_{0}^{2}}{\left[\left(1+\beta_{e} \beta_{i}\right)^{2}+\beta_{e}^{2}\right]}\left[\left(1+\beta_{e} \beta_{i}\right) v+\beta_{e} u\right], \\
\left(\rho c_{p}\right)_{n f}\left(u \frac{\partial T}{\partial x}+v \frac{\partial T}{\partial y}+w \frac{\partial T}{\partial z}\right)=k_{n f} \frac{\partial^{2} T}{\partial z^{2}}+\frac{\partial q_{r}}{\partial z}+Q^{\prime \prime \prime}+\frac{\sigma_{n f} B_{0}^{2}}{\left[\left(1+\beta_{e} \beta_{i}\right)^{2}+\beta_{e}^{2}\right]}\left(u^{2}+v^{2}\right), \\
u \frac{\partial a}{\partial x}+v \frac{\partial a}{\partial y}+w \frac{\partial a}{\partial z}=D_{A} \frac{\partial^{2} a}{\partial z^{2}}-k_{c} a b^{2}, \\
u \frac{\partial b}{\partial x}+v \frac{\partial b}{\partial y}+w \frac{\partial b}{\partial z}=D_{B} \frac{\partial^{2} b}{\partial z^{2}}-k_{c} a b^{2},
\end{gathered}
$$

where $(u, v, w)$ are the $(x, y, z)$ components of velocity, $k_{c}$ and $k_{e}$ are rate constants, $(A$, $B)$ are the rate of chemical species having concentration $(a, b), T$ is the temperature of fluid, $\rho$ is the fluid density, $\sigma$ is the fluid electrical conductivity, $D_{A}, D_{B}$ are the diffusion coefficients, $\beta_{e}$ is the Hall parameter and $\beta_{i}$ is the ion slip parameter respectively. $q_{r}$ is the thermal radiation flux and is given by the Stefan-Boltzmann law is

$$
q_{r}=-\frac{4 \sigma^{*}}{3 k^{*}} \frac{\partial T^{4}}{\partial z}=-\frac{16 \sigma^{*} T^{3}}{3 k^{*}} \frac{\partial T}{\partial z},
$$

where $\sigma^{*}$ and $k^{*}$ is Stefan-Boltzmann and Rosseland mean absorption coefficient, $T_{\infty}$ is the ambient temperature. Nonlinear heat source/sink $Q^{\prime \prime \prime}=\left(k_{f} u_{w} /(x+y)^{n} \nu_{f}\right)$

$$
\left[I_{1} *\left(T_{w}-T_{\infty}\right) f^{\prime}(\eta)+I_{2} *\left(T-T_{\infty}\right)\right] \text { is taken in the fourth coming analysis. Where } I_{1}
$$
and $I_{2}$ are heat generation/absorption coefficients respectively, 
To solve above set of governing equations, following boundary conditions are implemented

$$
\begin{gathered}
u=u_{w}=c(x+y)^{n}, v=v_{w}=d(x+y)^{n}, w=0, T=T_{w} \\
D_{A} \frac{\partial a}{\partial z}=k_{e} a, D_{B} \frac{\partial b}{\partial z}=-k_{e} a \text { at } z=0 \\
u \rightarrow 0, v \rightarrow 0, a \rightarrow a_{0}, T \rightarrow T_{\infty}, b \rightarrow 0 \quad \text { as } \quad z \rightarrow \infty
\end{gathered}
$$

In order to normalize the Eqs. (9)-(7), following changes of variable is suggested.

$$
\left.\begin{array}{c}
u=c(x+y)^{n} f^{\prime}(\eta), v=c(x+y)^{n} g^{\prime}(\eta), \eta=\sqrt{\frac{c(n+1)}{2 \nu_{f}}}(x+y)^{\frac{n-1}{2}} \\
w=-\sqrt{\frac{n+1}{2} \nu_{f} c(x+y)^{n-1}}\left[(f+g)+\left(\frac{n-1}{n+1}\right) \eta\left(f^{\prime}+g^{\prime}\right)\right], \\
T=T_{\infty}+\left(T_{w}-T_{\infty}\right) \theta(\eta), a=a j(\eta), b=b s(\eta)
\end{array}\right\}
$$

The correlations for effective density $\left(\rho_{n f}\right)$, effective viscosity $\left(\mu_{n f}\right)$, effective electrical conductivity $\left(\sigma_{n f}\right)$ and effective thermal conductivity $\left(k_{n f}\right)$ are [17] given by

$$
\begin{gathered}
\rho_{n f}=(1-\varphi) \rho_{f}+\varphi \rho_{s}, \quad\left(\rho c_{p}\right)_{n f}=(1-\varphi)\left(\rho c_{p}\right)_{f}+\varphi\left(\rho c_{p}\right)_{s}, \\
\sigma_{n f}=\sigma_{f}\left(1+\frac{3(\sigma-1) \varphi}{\sigma+2-(\sigma-1) \varphi}\right), \sigma=\frac{\sigma_{s}}{\sigma_{f}}, k_{n f}=\frac{k_{s}+2 k_{f}-2 \varphi\left(k_{f}-k_{s}\right)}{k_{s}+2 k_{f}+\varphi\left(k_{f}-k_{s}\right)} k_{f},
\end{gathered}
$$

\begin{tabular}{|c|c|c|c|c|c|}
\hline Thermal property & base fluid & $\mathrm{Fe}_{3} \mathrm{O}_{4}$ & $A g$ & $\mathrm{Al}_{2} \mathrm{O}_{3}$ & $\mathrm{TiO}_{2}$ \\
\hline$\rho /\left(k g \cdot m^{-3}\right)$ & 997.1 & 5180 & 10500 & 3970 & 4250 \\
\hline$c p /\left(J \cdot k g^{-1} \cdot K^{-1}\right)$ & 4179 & 670 & 235 & 765 & 686.2 \\
\hline$k /\left(W \cdot m^{-1} \cdot K^{-1}\right)$ & 0.613 & 9.7 & 429 & 40 & 8.9538 \\
\hline$\varphi$ & 0.00 & 0.05 & 0.10 & 0.15 & 0.20 \\
\hline$\sigma /\left(s \cdot m^{-1}\right)$ & 0.05 & 25000 & $6.6 \times 10^{-7}$ & $35 \times 10^{6}$ & $2.6 \times 10^{6}$ \\
\hline $\begin{array}{r}\left(\frac{1}{\varphi_{1}}\right) f^{\prime \prime \prime}- \\
H a( \\
f(0)=\end{array}$ & $\begin{array}{l}\frac{2 n}{2+1}\left(f^{\prime}+g^{\prime}\right) \\
\frac{1}{\left.\left.+\beta_{e} \beta_{i}\right)^{2}+\beta_{e}^{2}\right]}\end{array}$ & $\begin{array}{l}\mathrm{C}^{(1+} \\
f^{\prime}(\infty)\end{array}$ & 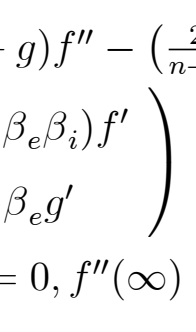 & $\begin{array}{l}\left.\frac{2}{+1}\right)\left(\frac{\varphi_{2}}{\varphi_{1}}\right) \\
=0,\end{array}$ & , \\
\hline
\end{tabular}

where $\rho, k, \sigma, \varphi$ and $c_{p}$, respectively, are density, thermal conductivity, electrical conductivity, volume fraction and specific heat. The subscripts $f, n f$ and $s$ stands for fluid, nanofluid and solid particles ( nano-particles) respectively.

Table 1. Thermo-physical properties of water and nano-particles. 


$$
\left.\begin{array}{c}
\left(\frac{1}{\varphi_{1}}\right) g^{\prime \prime \prime}-\frac{2 n}{n+1}\left(f^{\prime}+g^{\prime}\right) g^{\prime}+(f+g) g^{\prime \prime}-\left(\frac{2}{n+1}\right)\left(\frac{\varphi_{2}}{\varphi_{1}}\right) \\
H a\left(\frac{1}{\left[\left(1+\beta_{e} \beta_{i}\right)^{2}+\beta_{e}^{2}\right]}\right)\left(\begin{array}{c}
\left(1+\beta_{e} \beta_{i}\right) g^{\prime} \\
+\beta_{e} f^{\prime}
\end{array}\right)=0, \\
g(0)=0, g(\infty)=\alpha,
\end{array}\right\},
$$

where $H a$ is the Hartmann number, $E c$ is the Eckert number, $\operatorname{Pr}$ is the Prandtl number, $\alpha$ is the velocity ration parameter, $\theta_{w}$ temperature parameter, $N_{R}$ is the radiation parameter, $K^{*}$ is the homogeneous reaction strength, $K^{s}$ is the heterogeneous reaction strength, $S c$ is the Schmidt number and $\varepsilon$ is the Diffusion ratio coefficient.

If diffusion coefficients for chemical species are same then $\varepsilon=1$ which yields

$$
\begin{gathered}
j(\eta)+s(\eta)=1, \\
\left(\frac{1}{S c}\right) j^{\prime \prime}+(f+g) j^{\prime}-\frac{2}{n+1} K^{*} j(1-j)^{2}=0, \\
j^{\prime}(0)=\sqrt{\frac{2}{n+1}} K^{s} j(0), j(\infty)=1,
\end{gathered}
$$

The expressions for above dimensionless problems are

$$
\begin{gathered}
H a=\frac{\sigma_{f} B_{0}^{2}}{\rho_{f} c}, \operatorname{Pr}=\frac{\left(\mu c_{p}\right)_{f}}{k_{f}}, E c=\frac{c(x+y)^{n+1}}{\left(c_{p}\right)_{f}\left(T_{w}-T_{\infty}\right)}, N_{R}=\frac{3 k_{f} k^{*}}{16 \sigma^{*} T_{\infty}^{3}}, \alpha=\frac{d}{c}, \theta_{w}=\frac{T_{w}}{T_{\infty}}, \\
, \varepsilon=\frac{D_{B}}{D_{A}}, S c=\frac{\mu_{f}}{\rho_{f} D_{A}}, K^{*}=\frac{k_{c} a}{c(x+y)^{n-1}}, K^{s}=\frac{k_{e}}{D_{A}} \sqrt{\frac{\nu_{f}}{c(x+y)^{n-1}}} .
\end{gathered}
$$

Normalized wall shear stress (skin friction coefficient $C_{f}, C_{g}$ ) and normalized wall heat flux (Nusselt number $N u$ ), respectively, are 


$$
\begin{gathered}
C_{f}=\frac{\left.\mu_{n f}\left(\frac{\partial u}{\partial z}+\frac{\partial w}{\partial x}\right)\right|_{z=0}}{\frac{1}{2} \rho_{f}\left(u_{w}\right)^{2}}=\frac{1}{\sqrt{\operatorname{Re}}} \frac{1}{(1-\varphi)^{2.5}} f^{\prime \prime}(0), \\
C_{g}=\frac{\left.\mu_{n f}\left(\frac{\partial v}{\partial z}+\frac{\partial w}{\partial y}\right)\right|_{z=0}}{\frac{1}{2} \rho_{f}\left(u_{w}\right)^{2}}=\frac{1}{\sqrt{\operatorname{Re}}} \frac{1}{(1-\varphi)^{2.5}} g^{\prime \prime}(0), \\
N u=-\frac{\left.(x+y) k_{n f} \mu_{n f} \frac{\partial T}{\partial z}\right|_{z=0}=-\sqrt{\operatorname{Re}}\left[1+\frac{4}{3 N_{R}}\left(1+\left(\theta_{w}-1\right)\right)[\theta(0)]^{2}\right] \theta^{\prime}(0),}{k_{n f}\left(T_{w}-T_{\infty}\right)}=
\end{gathered}
$$

where $\operatorname{Re}=c(x+y)^{2} / \nu_{f}$ and is the local Reynolds number.

\section{Numerical procedure}

$$
\begin{aligned}
& K_{i j}^{11}=\int_{\eta_{e}}^{\eta_{e+1}} \psi_{i} \frac{d \psi_{j}}{d \eta} d \eta, K_{i j}^{13}=-\int_{\eta_{e}}^{\eta_{e+1}} \psi_{i} \psi_{j} d \eta, K_{i j}^{12}=0, K_{i j}^{14}=0, K_{i j}^{15}=0, K_{i j}^{16}=0 \\
& K_{i j}^{22}=\int_{\eta_{e}}^{\eta_{e+1}} \psi_{i} \frac{d \psi_{j}}{d \eta} d \eta, K_{i j}^{24}=-\int_{\eta_{e}}^{\eta_{e+1}} \psi_{i} \psi_{j} d \eta, K_{i j}^{21}=0, K_{i j}^{23}=0, K_{i j}^{25}=0, K_{i j}^{26}=0 \\
& K_{i j}^{31}=\int_{\eta_{e}}^{\eta_{e+1}}\left[\begin{array}{c}
\left(\frac{1}{\varphi_{1}}\right) \frac{d \psi_{i}}{d \eta} \frac{d \psi_{j}}{d \eta}-\frac{2 n}{n+1}(\bar{h}+\bar{k}) \psi_{i} \psi_{j}+(\bar{f}+\bar{g}) \psi_{i} \frac{d \psi_{j}}{d \eta} \\
-\left(\frac{2}{n+1}\right)\left(\frac{\varphi_{2}}{\varphi_{1}}\right) H a\left(\frac{\left(1+\beta_{e} \beta_{i}\right)}{\left[\left(1+\beta_{e} \beta_{i}\right)^{2}+\beta_{e}^{2}\right]}\right) \psi_{i} \psi_{j}
\end{array}\right] d \eta \\
& K_{i j}^{33}=\int_{\eta_{e}}^{\eta_{e+1}}\left(\frac{2}{n+1}\right)\left(\frac{\varphi_{2}}{\varphi_{1}}\right) H a\left(\frac{\beta_{e}}{\left[\left(1+\beta_{e} \beta_{i}\right)^{2}+\beta_{e}^{2}\right]}\right) \psi_{i} \psi_{j} d \eta \\
& K_{i j}^{32}=0, K_{i j}^{34}=0, K_{i j}^{35}=0, K_{i j}^{36}=0 \\
& K_{i j}^{44}=\int_{\eta_{e}}^{\eta_{e+1}}\left[\begin{array}{c}
\left(\frac{1}{\varphi_{1}}\right) \frac{d \psi_{i}}{d \eta} \frac{d \psi_{j}}{d \eta}-\frac{2 n}{n+1}(\bar{h}+\bar{k}) \psi_{i} \psi_{j}+(\bar{f}+\bar{g}) \psi_{i} \frac{d \psi_{j}}{d \eta} \\
-\left(\frac{2}{n+1}\right)\left(\frac{\varphi_{2}}{\varphi_{1}}\right) H a\left(\frac{\left(1+\beta_{e} \beta_{i}\right)}{\left[\left(1+\beta_{e} \beta_{i}\right)^{2}+\beta_{e}^{2}\right]}\right) \psi_{i} \psi_{j}
\end{array}\right] d \eta \\
& K_{i j}^{43}=\int_{\eta_{e}}^{\eta_{e+1}}-\left(\frac{2}{n+1}\right)\left(\frac{\varphi_{2}}{\varphi_{1}}\right) H a\left(\frac{\beta_{e}}{\left[\left(1+\beta_{e} \beta_{i}\right)^{2}+\beta_{e}^{2}\right]}\right) \psi_{i} \psi_{j} d \eta \\
& K_{i j}^{41}=0, K_{i j}^{42}=0, K_{i j}^{45}=0, K_{i j}^{46}=0, \\
& K_{i j}^{53}=\int_{\eta_{e}}^{\eta_{e+1}}\left[\left(\frac{1}{\varphi_{3}}\right)\left(\frac{2}{n+1}\right) I_{1} \psi_{i} \psi_{j}+\frac{\mu_{n f}}{\mu f} \frac{\varphi_{2}}{\varphi_{3}} H a \operatorname{Pr} E c\left(\frac{1}{\left[\left(1+\beta_{e} \beta_{i}\right)^{2}+\beta_{e}^{2}\right]}\right) \bar{h} \psi_{i} \psi_{j}\right] d \eta \\
& K_{i j}^{54}=\int_{\eta_{e}}^{\eta_{e+1}}\left[\frac{\mu_{n f}}{\mu f} \frac{\varphi_{2}}{\varphi_{3}} H a \operatorname{Pr} E c\left(\frac{1}{\left[\left(1+\beta_{e} \beta_{i}\right)^{2}+\beta_{e}^{2}\right]}\right) \bar{k} \psi \psi_{j}\right] d \eta, K_{i j}^{51}=0, K_{i j}^{52}=0, K_{i j}^{56}=0,
\end{aligned}
$$




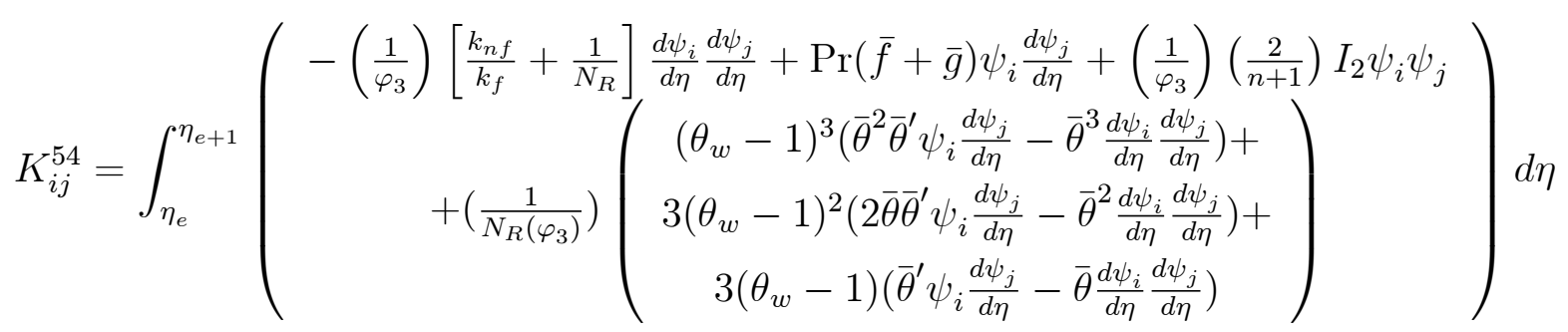

$$
\begin{aligned}
& K_{i j}^{66}=\int_{\eta_{e}}^{\eta_{e+1}}\left[-\left(\frac{1}{S c}\right) \frac{d \psi_{i}}{d \eta} \frac{d \psi_{j}}{d \eta}+(f+g) \psi_{i} \frac{d \psi_{j}}{d \eta}-\frac{2}{n+1} K^{*}(1-\bar{j})^{2} \psi_{i} \psi_{j}\right] d \eta, \\
& K_{i j}^{61}=0, K_{i j}^{62}=0, K_{i j}^{63}=0, K_{i j}^{64}=0, K_{i j}^{65}=0, \\
& b_{i}^{3}=-\int_{\Gamma}\left(\frac{1}{\varphi_{1}}\right) \psi_{i} \frac{d h}{d \eta}, b_{i}^{4}=-\int_{\Gamma}\left(\frac{1}{\varphi_{1}}\right) \psi_{i} \frac{d k}{d \eta}, b_{i}^{6}=-\int_{\Gamma}\left(\frac{1}{S c}\right) \psi_{i} \frac{d \varphi}{d \eta}, \\
& b_{i}^{5}=-\int_{\Gamma}\left(\left(\frac{1}{\varphi_{3}}\right)\left[\frac{k_{n f}}{k_{f}}+\frac{1}{N_{R}}\right] \psi_{i} \frac{d \theta}{d \eta}++\left(\frac{1}{N_{R}\left(\varphi_{3}\right)}\right)\left(\begin{array}{c}
\left(\theta_{w}-1\right)^{3} \bar{\theta}^{3} \psi_{i} \frac{d \theta}{d \eta}+ \\
3\left(\theta_{w}-1\right)^{2} \bar{\theta}^{2} \psi_{i} \frac{d \theta}{d \eta}+ \\
\left.3\left(\theta_{w}-1\right) \bar{\theta} \psi_{i} \frac{d \theta}{d \eta}\right)
\end{array}\right)\right)
\end{aligned}
$$

where $\bar{f}=\sum \psi_{j} \bar{f}_{j}, \bar{h}=\sum \psi_{j} \bar{h}_{j}, g=\sum \psi_{j} g_{j}, \bar{k}=\sum \psi_{j} \bar{k}_{j}, \bar{\theta}=\sum \psi_{j} \bar{\theta}_{j}$ and $\bar{\varphi}=\sum \psi_{j} \bar{\varphi}$. $\bar{f}, \bar{h}, \bar{\theta}$ and $\bar{\varphi}$ are nodal values at previous iteration.

\section{Results and discussion}

Simultaneous effects of homogeneous, heterogeneous chemical reactions in magnetized and partially ionized nano-liquid in the presence of dissipation phenomenon (during thermal changes) are investigated theoretically. The mathematical models are solved numerically using a powerful technique FEM. The various effects are simulated through numerical experiments. Flow fluids, wall shear stresses, wall heat flux and mass flux under some emerging physical parameters are analyzed. These observations noted during numerical experiments are displayed graphically and in the form of numerical data.

Fluid flow behavior: The effects of Hall parameter $\left(\beta_{e}\right)$, ion slip parameter $\left(\beta_{i}\right)$, powerindex $(n)$, stretching ratio parameter $(\alpha)$, Hartmann number $(H a)$, nanosized metallic particles on $x$ - and $y$-components of the velocity are displayed in Fig 1-12. Fig. 1 clearly depicts that an increase in Hall parameter $\beta_{e}\left(=\omega_{e} \tau_{e}\right)$ causes an enhancement in the movement of magnetized partially ionized liquid in $x$-direction. The Hall parameter $\beta_{e}\left(=\omega_{e} \tau_{e}\right)$ is the product of frequency of electrons and electron collision time and an increase in Hall 
parameter $\beta_{e}$ implies either an increase in the frequency of electrons or electrons collision time or both frequency and collision time due to which Hall current results a force called Hall force. It is experimentally verified that Hall force is opposite to magnetic force. Here in this case, magnetic force opposes the flow. Therefore, an increase in the velocity of the fluid in $x$-direction due to an increase in Hall parameter $\beta_{e}$ is expected and Fig. 1 depicts the same physics as it is expected. Hence present theoretical results are completely in line with under lying physics. During numerical simulations, boundary layer thickness is observed to increase when Hall parameter $\beta_{e}$ is increased. It is also noted that the ion-slip parameter $\beta_{i}\left(=\omega_{i} \tau_{i}\right)$ has effect on $x$-component of velocity similar to Hall parameter $\beta_{e}$ on $x$-component of partially ionized liquid. This behavior of $x$-component of velocity under the variation of ion-slip parameter $\beta_{i}$ is shown by Fig. 2. The behaviors of power-index and ratio of stretching rates of elastic sheet on the $x$-component of velocity are shown by Figs. 3 and 4 respectively. The $x$-component of velocity decreases when power-index of stretching velocities of elastic sheet is increased (see Fig. 3). Fig. 4 displays the behavior of $x$-component of velocity of partially ionized liquid when $\alpha(=d / c)$ is less than unity. Therefore, it is concluded that $x$-component of velocity decreases when the stretching rate of elastic sheet in $y$-direction is greater than the stretching rate in $x$-direction. The effect of variation of intensity of magnetic field on the flow of partially ionized liquid in $x$-direction is displayed by Fig. 5 and 11 which show that fluid experiences an opposing magnetic force and consequently, flow slows down when intensity of magnetic field is increased. A significant reduction in boundary layer thickness is observed due to an increase in the intensity of magnetic field. The effect of nano-particles $\mathrm{Fe}_{3} \mathrm{O}_{4}, \mathrm{Ag}, \mathrm{Al}_{2} \mathrm{O}_{3}$ and $\mathrm{TiO}_{2}$ on the transport momentum is given by Fig. 6 and 12. In Fig. 6 the effect of $\varphi$ on $x$-component of velocity of $\mathrm{TiO}_{2}$ nano-liquid is greater than the velocity of $\mathrm{Ag}$ nano-liquid, $\mathrm{Fe}_{3} \mathrm{O}_{4}$ nano-liquid and $\mathrm{Al}_{2} \mathrm{O}_{3}$ nano-liquid. The influence of Hall and ion slip parameters $\beta_{e}$ and $\beta_{i}$ on $y$-component of velocity of partially ionized liquid is demonstrated by Figs. 7 and 8 respectively. It is observed that the effects of Hall and ion-slip parameters $\beta_{e}$ and $\beta_{i}$ on $x$-component of velocity of nano-liquid are similar. The impact of power-index of wall velocity on $y$-component of velocity is shown in fig. 9 . $y$-component of velocity has increasing trend when power-index of wall velocity is increased. A notable increase in $y$-component of velocity is observed when $\alpha$ (the ratio of stretching rates) is increased is shown in Fig. 10. Momentum diffuses in $y$-direction is faster than diffusion of momentum in $x$-direction. 
Heat transfer observations: The impact of Hall and ion slip parameters on the temperature of partially ionized liquid is simulated in Figs. 13 and 14. Hall and ion slip parameters $\left(\beta_{e}, \beta_{i}\right)$ appear in denominator of term (in energy Eq. (4)) due to Ohmic dissipation. Therefore an increase in Hall and ion slip parameters, reduce the dissipation of heat and therefore temperature of nano-liquid is decreased. Thermal boundary layer thickness associated with ionized MHD liquid is less than that associated with hydro dynamic slow. The behavior of power-law index $n$ of wall velocity on the temperature of nano-liquid is simulated and the resulting temperature curves are shown in Fig. 15. It is founded from Fig. 15 that temperature of ionized nano-liquid decreases when power-law index $n$ is increased. This decreased in temperature is obvious because power law index $n$ appears in the denominator of heat generation terms in energy Eq. 13 and an increase in $n$ slows down heat generation. Consequently, temperature decreases. The parameter $\alpha$ is the ratio of stretching rate $d$ in $y$-direction to the stretching rate $c$ in $x$-direction and influence of this stretching rates parameter $\alpha$ is shown in Fig. 16 This graphical representation in Fig. 16 depicts that for $d<c$, temperature has a decreasing trend and so is thermal boundary layer thickness. The intensity of the magnetic field has profound effect on the temperature of nano-fluid. The Ohmic dissipation is directly proportional to the square of the magnitude of current density and current density is proportional to the velocity of fluid and intensity of magnetic field. This implies that the Ohmic dissipation is directly proportional to the intensity of magnetic field. Therefore, an increase in Ohmic dissipation with an increase in magnetic intensity is expected. Amazingly, present theoretical results depict the same physics as is expected. Further, the heated dissipates due to Joule heating adds to the fluid and consequently, temperature of the fluid increases as shown in Fig. 17. Different type of nanosized metallic particles are dispersed in partially ionized liquid. This dispersion of nanosized particles increases the effective thermal conductivity of the mixture, the mixture of nano-particles and base fluid and resultant the process of heat transfer is enhanced due to which fluid temperature of ionized liquid rises. This fact is displayed in Fig. 18. It is also observed that mixture of base fluid and $\mathrm{Fe}_{3} \mathrm{O}_{4}$ nanoparticle has highest effective thermal conductivity as compare to the effective thermal conductivity of mixture containing $\mathrm{Ag}, \mathrm{Al}_{2} \mathrm{O}_{3}$ and $\mathrm{TiO}_{2}$ nano-particles. Hence, the dispersion of $\mathrm{Ag}_{2} \mathrm{Al}_{2} \mathrm{O}_{3}$ and $\mathrm{TiO}_{2}$ nano-particles in nano-liquid is recommended for the highest thermal efficiency. The partially ionized liquid is assumed to be thermally radiative. The effect of thermal radiations on the temperature of partially ionized liquid is examined theoretically and consequent 
temperature curves are displayed in Fig. 19. Electromagnetic waves carry thermal energy away from liquid regime which results a significant decrease in temperature of the ionized liquid. The behavior of temperature field under non-uniform heat generation is sketched in Fig. 20. Non-uniform heat generation results a significant increase in temperature of ionized liquid. The effect of non-uniform heat generation on temperature of chemical reaction species is shown in Fig. 21. The heat generates as a result of convection and thermal changes in the fluid adds to the fluid partially ionized fluid and as a consequent of this, temperature of fluid rises. The effects of temperature parameter $\theta_{w}$ on the dimensionless temperature of partially ionized fluid are simulated and obtained simulated results are represented by the temperature curves shown in Fig. 22. The temperature parameter $\theta_{w}\left(=T_{w} / T_{\infty}\right)$ is the ratio of wall temperature $T_{w}$ to the ambient temperature $T_{\infty}$ and an increase temperature parameter $\theta_{w}$ corresponds to a decrease in the ambient temperature. An increase in wall temperature and a decrease in ambient temperature cause a temperature difference. Due to this temperature differences, conduction of heat from wall to the fluid speeds up which results a rise in temperature. The present theoretical simulations depict exactly the same as under lying physics depicts.

Dynamics of concentration of reacting species: The dynamics of concentration field under the variation of the parameters $S c, n, K^{s}$ and $\varphi$ are sketched in Figs. 23-26. The concentration field decreases when Schmidt number $S c$ is increased. An opposite trend of concentration boundary layer thickness verses Schmidt number is noted (see Fig. 23). An increase in power-index of wall-velocity is responsible for a significant increase in the concentration boundary layer thickness. This is observation can be seen in Fig. 24. The influences of homogeneous chemical strength $K^{*}$ on the concentration is investigated and the behavior of concentration fluid is displayed in Fig. 25 which reflects that concentration field decreases as homogeneous chemical strength $K^{*}$ is increased. However concentration boundary layer thickness decreases with an increase of homogeneous chemical strength. The effect of heterogeneous chemically reacting species is analyzed and resulting simulations are displayed in Fig. 26. The comparison of Fig. 25 and 26 shows that homogeneous chemical reactions have similar effects on concentration field in qualitative sense. But in quantitative sense, the effect of homogeneous chemical reactions more significant as compare to the effect of heterogeneous chemical reaction. 


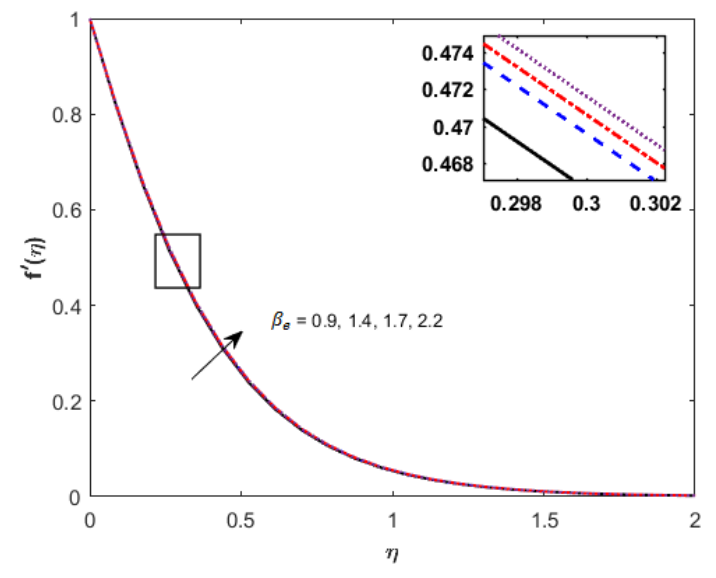

Fig. 1. Velocity $f^{\prime}$ verses $\beta_{e}$ when $n=1.1, \varphi=0.05, \alpha=0.1, H a=0.8$ and $\beta_{i}=0.1$.

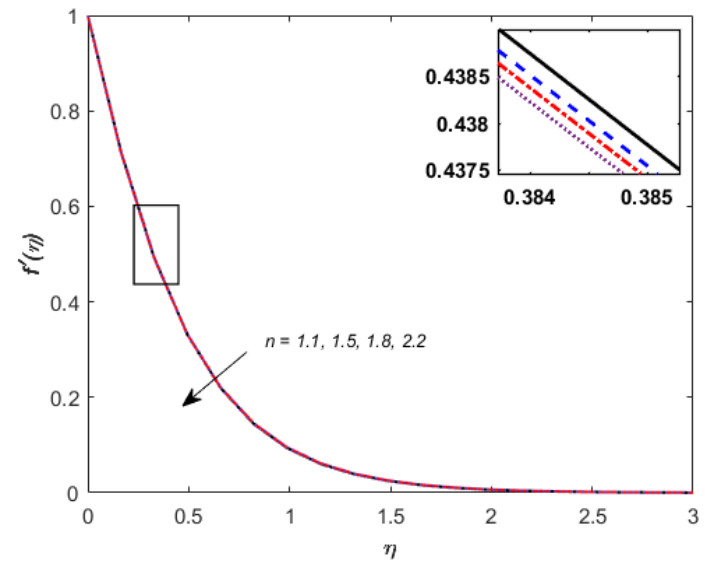

Fig. 3. Velocity $f^{\prime}$ verses $n$ when

$$
\begin{gathered}
\beta_{i}=0.1, \alpha=0.1, \varphi=0.05, H a=0.8 \text { and } \\
\beta_{e}=0.3 .
\end{gathered}
$$

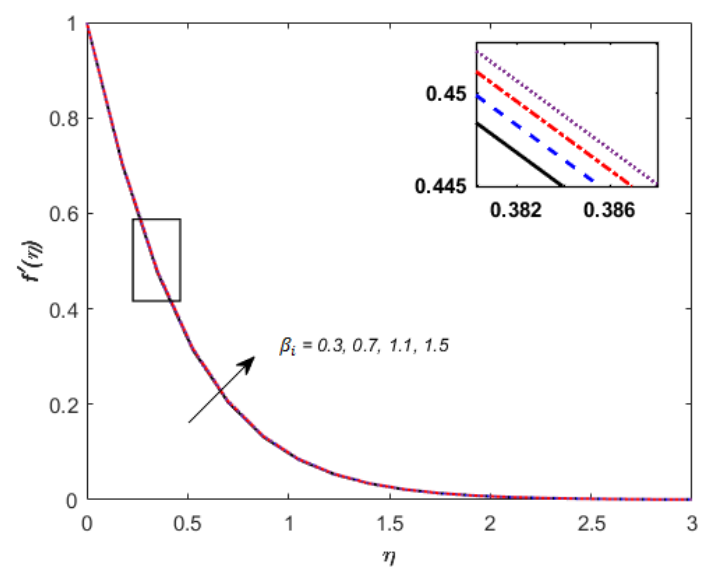

Fig. 2.Velocity $f^{\prime}$ verses $\beta_{i}$ when $n=1.1, \varphi=0.05, \alpha=0.1, H a=0.8$ and $\beta_{e}=0.3$.

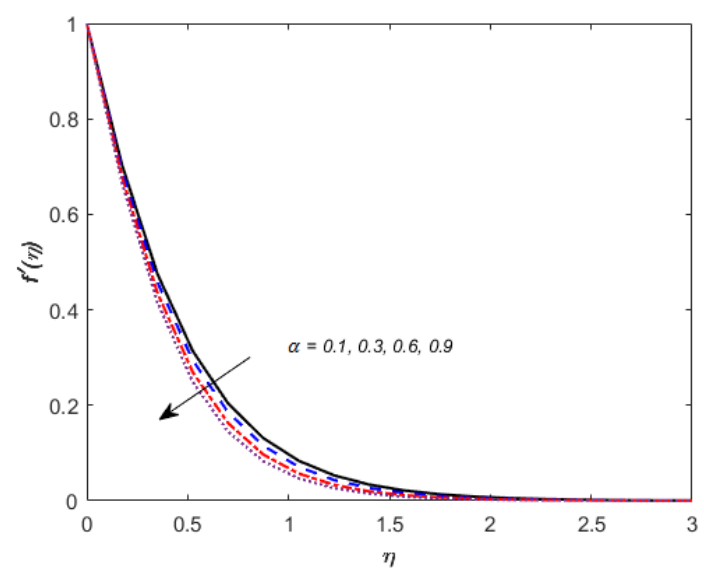

Fig. 4.Velocity $f^{\prime}$ verses $\alpha$ when $\beta_{i}=0.1, \varphi=0.05, n=1.1, H a=0.8$ and $\beta_{e}=0.3$. 


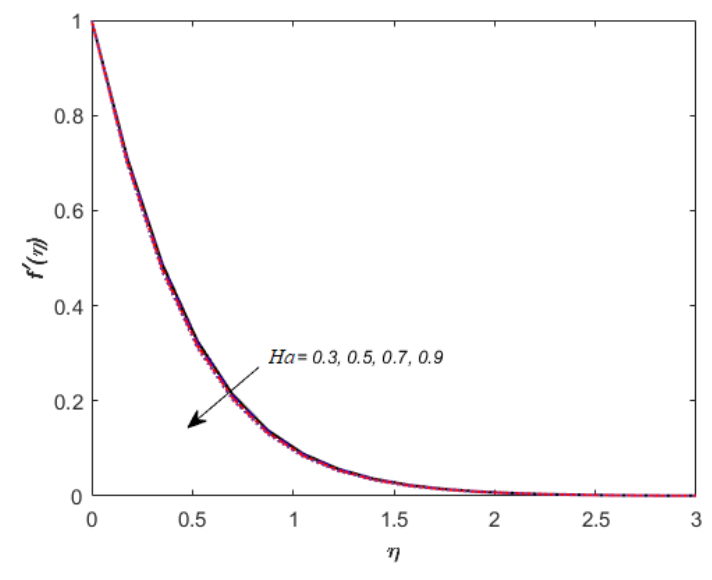

Fig. 5.Velocity $f^{\prime}$ verses $H a$ when

$$
\begin{gathered}
\beta_{i}=0.3, \varphi=0.05, \alpha=0.1, n=1.1 \text { and } \\
\beta_{e}=0.9 .
\end{gathered}
$$

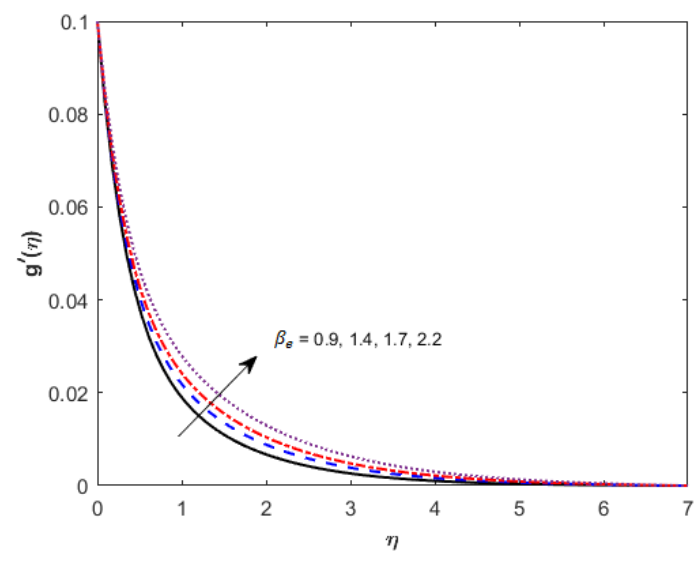

Fig. 7. Velocity $g^{\prime}$ verses $\beta_{e}$ when

$$
\begin{gathered}
n=1.1, \varphi=0.05, \alpha=0.1, H a=0.8 \text { and } \\
\beta_{i}=0.1 .
\end{gathered}
$$

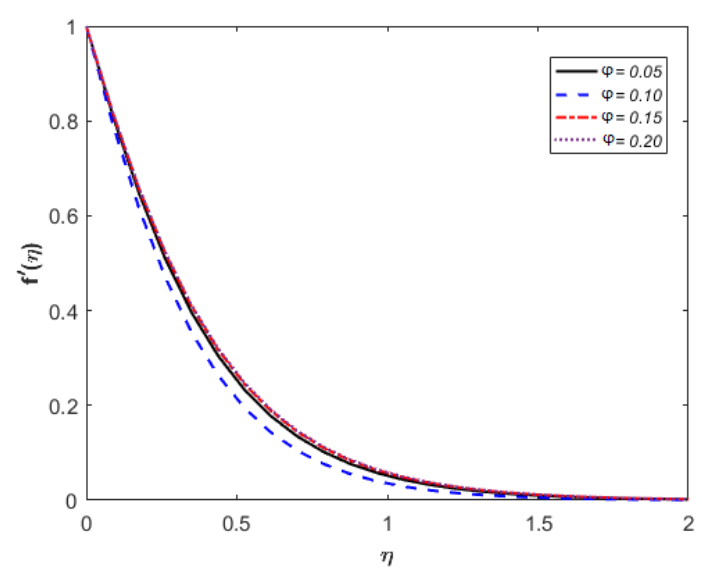

Fig. 6. Velocity $f^{\prime}$ verses $\varphi$ when

$$
\beta_{i}=0.1, n=1.1, \alpha=0.1, H a=0.8 \text { and }
$$$$
\beta_{e}=0.3 \text {. }
$$

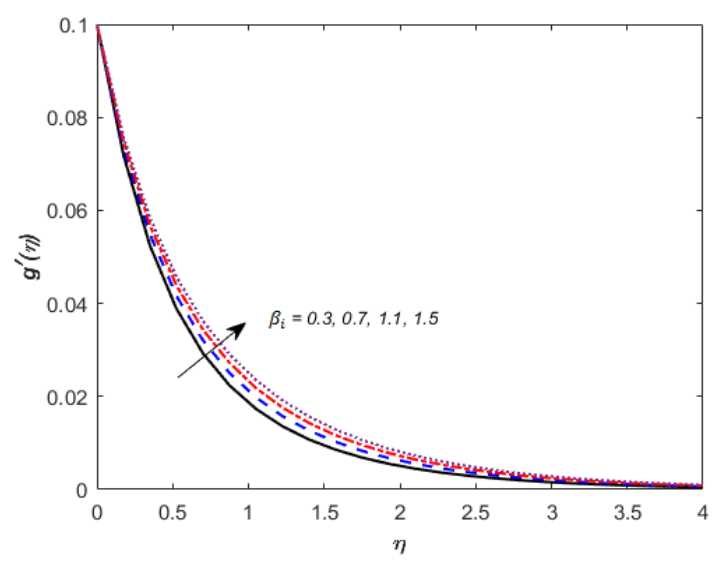

Fig. 8.Velocity $g^{\prime}$ verses $\beta_{i}$ when $n=1.1, \varphi=0.05, \alpha=0.1, H a=0.8$ and

$$
\beta_{e}=0.3 \text {. }
$$




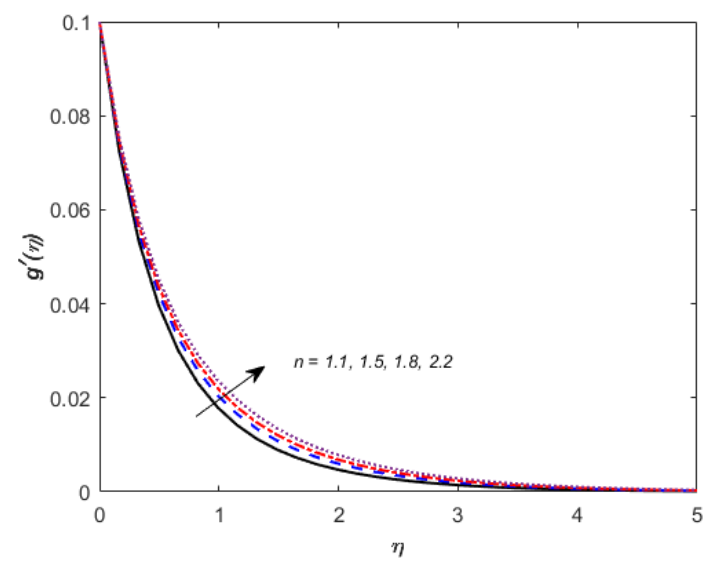

Fig. 9. Velocity $g^{\prime}$ verses $n$ when

$$
\begin{gathered}
\beta_{i}=0.1, \alpha=0.1, \varphi=0.05, H a=0.8 \text { and } \\
\beta_{e}=0.3 .
\end{gathered}
$$

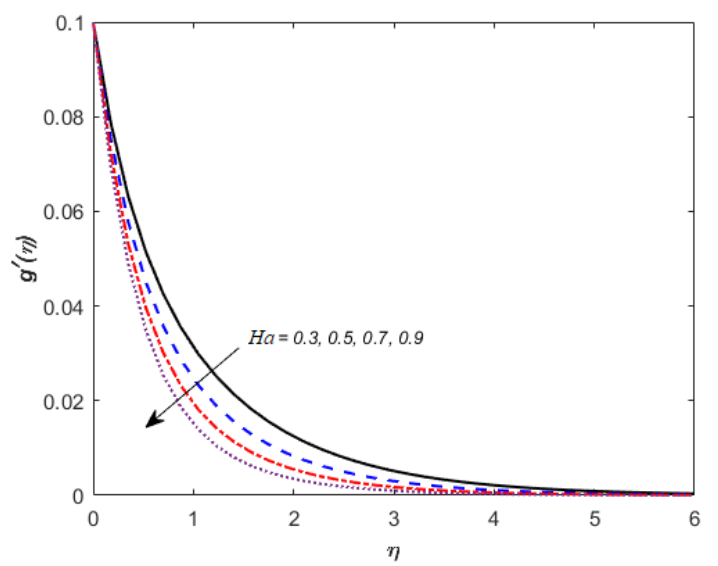

Fig. 11. Velocity $g^{\prime}$ verses $H a$ when

$$
\begin{gathered}
\beta_{i}=0.1, \varphi=0.05, \alpha=0.1, n=1.1 \text { and } \\
\beta_{e}=0.3 .
\end{gathered}
$$

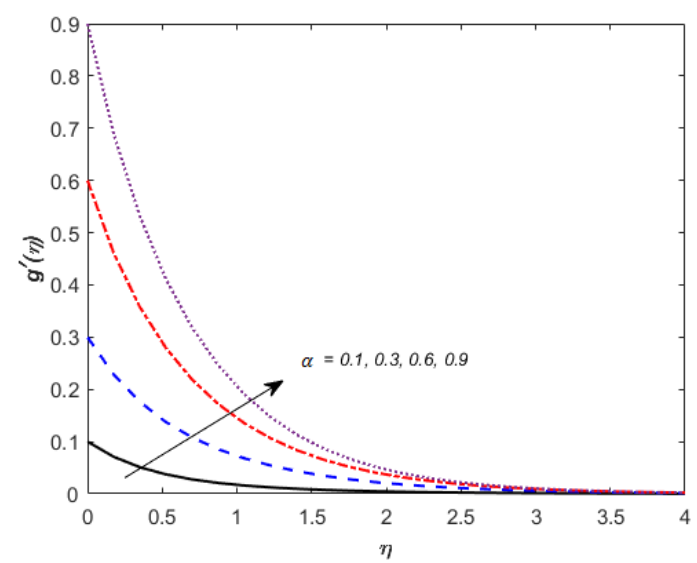

Fig. 10.Velocity $g^{\prime}$ verses $\alpha$ when $\beta_{i}=0.1, \varphi=0.05, n=1.1, H a=0.8$ and $\beta_{e}=0.3$.

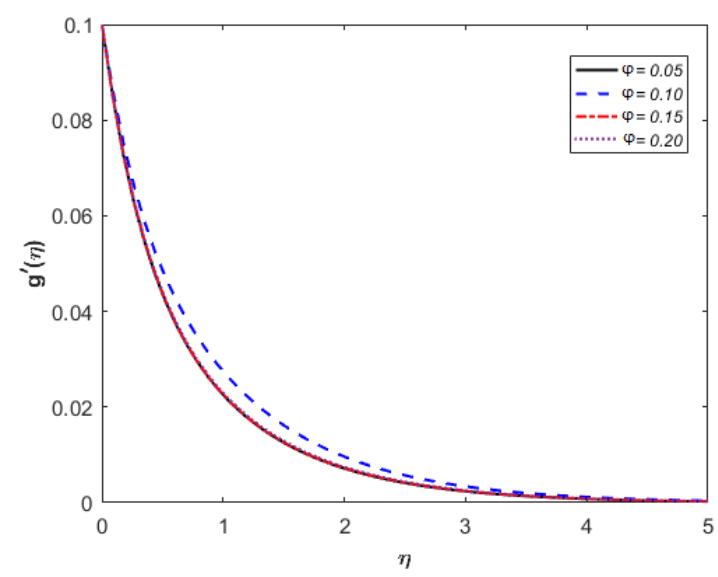

Fig. 12. Velocity $g^{\prime}$ verses $\varphi$ when $\beta_{i}=0.1, H a=0.8, \alpha=0.1, n=1.1$ and $\beta_{e}=0.3$. 


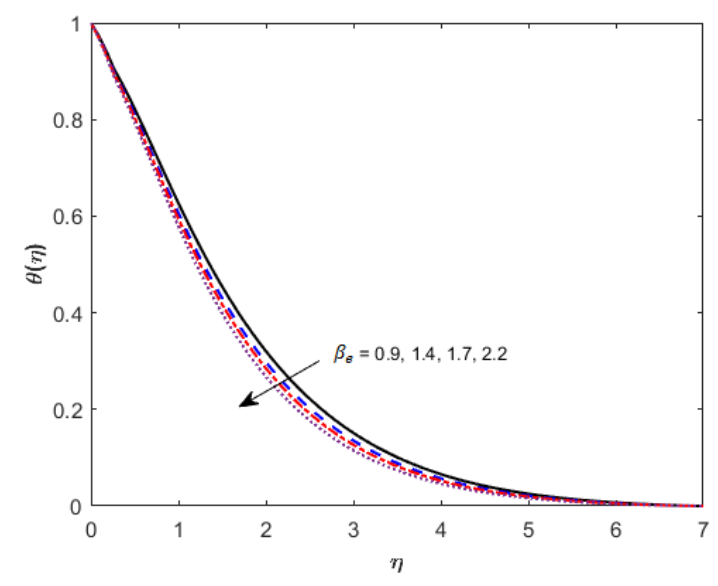

Fig. 13. Temperature $\theta$ verses $\beta_{e}$ when $n=1.1, \varphi=0.05, \operatorname{Pr}=7, E c=0.2, H a=$ $0.8, \beta_{i}=0.1, N_{R}=2.5, \theta_{w}=0.3, I_{1}=0.2$ and $I_{2}=0.4$.

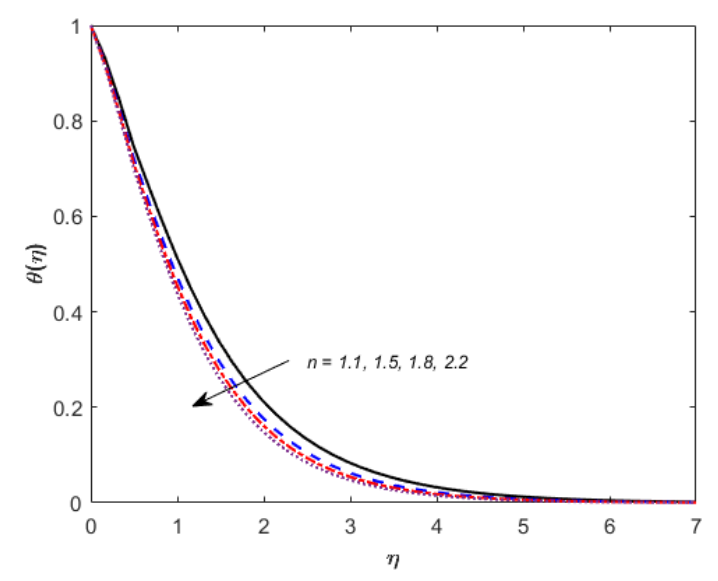

Fig. 15. Temperature $\theta$ verses $n$ when

$$
\begin{gathered}
\beta_{i}=0.1, \operatorname{Pr}=7, E c=0.2, \mathrm{Ha}=0.8, \beta_{e}= \\
0.3, N_{R}=2.5, \theta_{w}=0.3, I_{1}=0.2, I_{2}=0.4 \\
\text { and } \varphi=0.05 .
\end{gathered}
$$

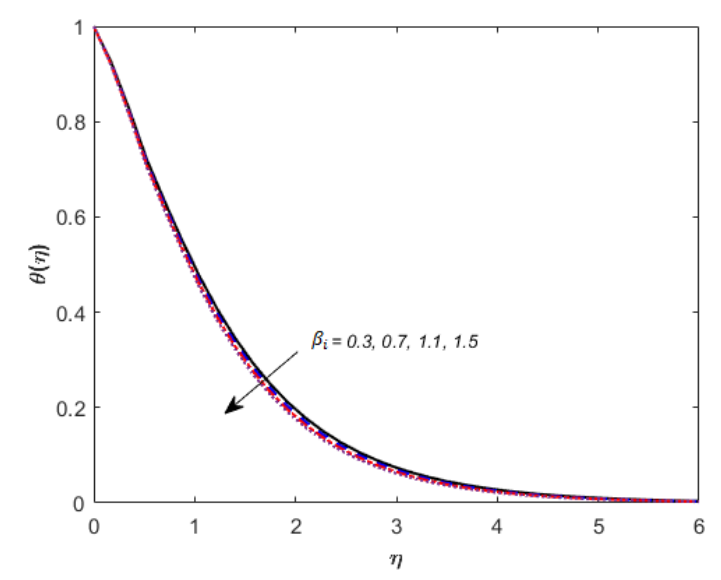

Fig. 14. Temperature $\theta$ verses $\beta_{i}$ when $n=1.1, \operatorname{Pr}=7, E c=0.2, H a=0.8, \beta_{e}=$ $0.3, N_{R}=2.5, \theta_{w}=0.3, I_{1}=0.2, I_{2}=0.4$ and $\varphi=0.05$.

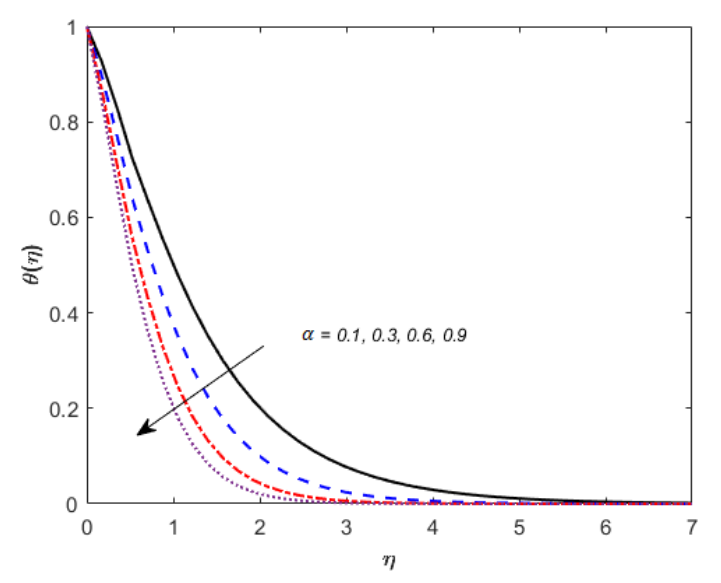

Fig. 16. Temperature $\theta$ verses $\alpha$ when $\beta_{i}=0.1, n=1.1, \operatorname{Pr}=7, E c=0.2, H a=$ $0.8, \beta_{e}=0.3, N_{R}=2.5, \theta_{w}=0.3, I_{1}=$ $0.2, I_{2}=0.4$ and $\varphi=0.05$. 


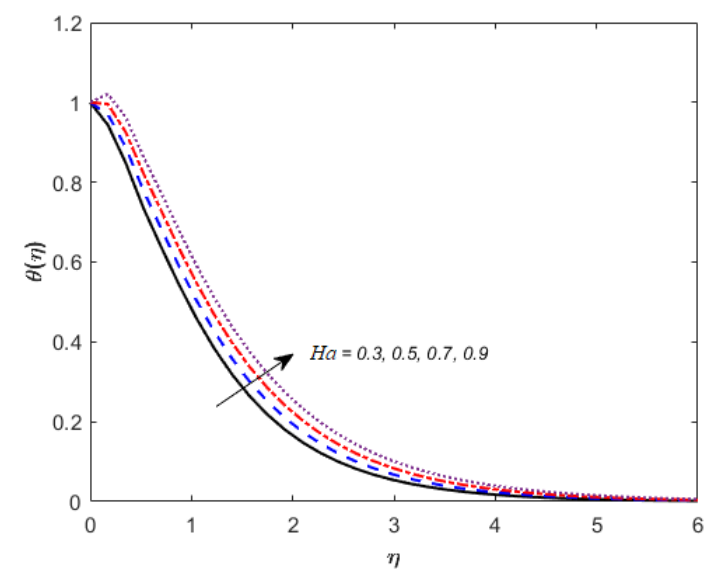

Fig. 17. Temperature $\theta$ verses $H a$ when $\beta_{i}=0.3, \operatorname{Pr}=7, E c=0.2, n=1.1, \beta_{e}=$ $0.9, N_{R}=2.5, \theta_{w}=0.3, I_{1}=0.2, I_{2}=0.4$ and $\varphi=0.05$.

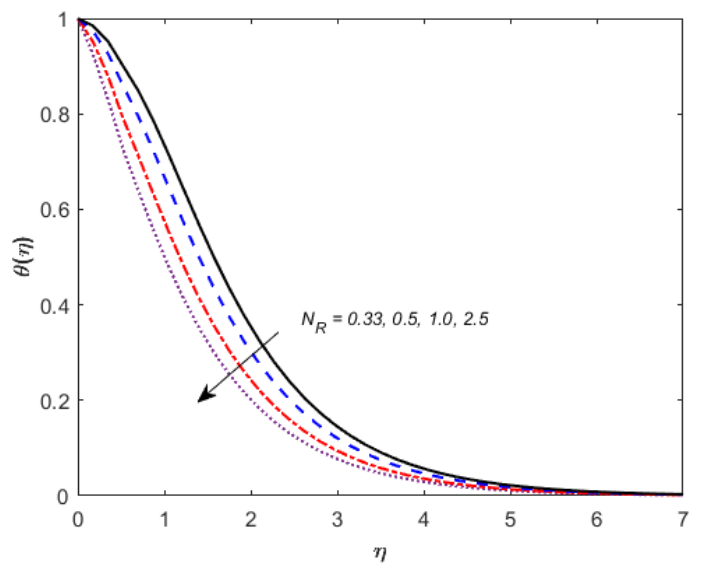

Fig. 19. Temperature $\theta$ verses $N_{R}$ when $\beta_{i}=0.1, \alpha=0.1, \operatorname{Pr}=3, E c=0.2, n=$ $1.1, \beta_{e}=0.3, H a=0.8, \theta_{w}=0.3, I_{1}=$ $0.2, I_{2}=0.4$ and $\varphi=0.05$.

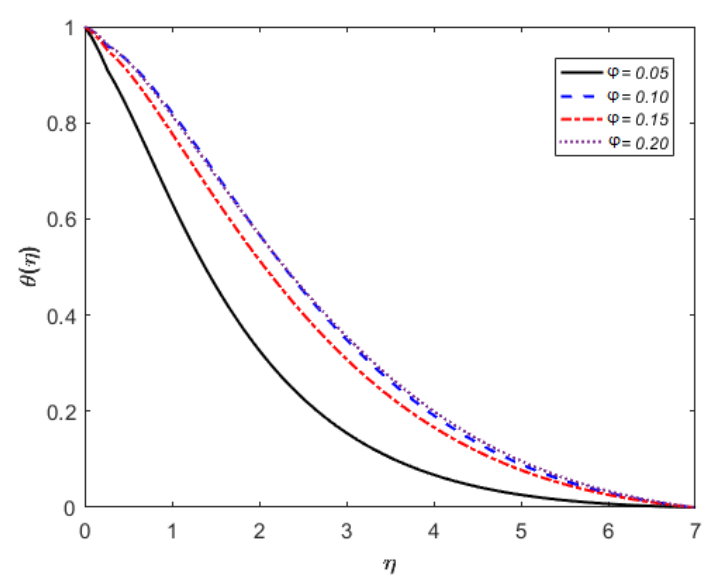

Fig. 18. Temperature $\theta$ verses $\varphi$ when $\beta_{i}=0.1, \operatorname{Pr}=3, E c=0.2, H a=0.8, \beta_{e}=$ $0.3, N_{R}=2.5, \theta_{w}=0.3, I_{1}=0.2, I_{2}=0.4$ and $n=1.1$.

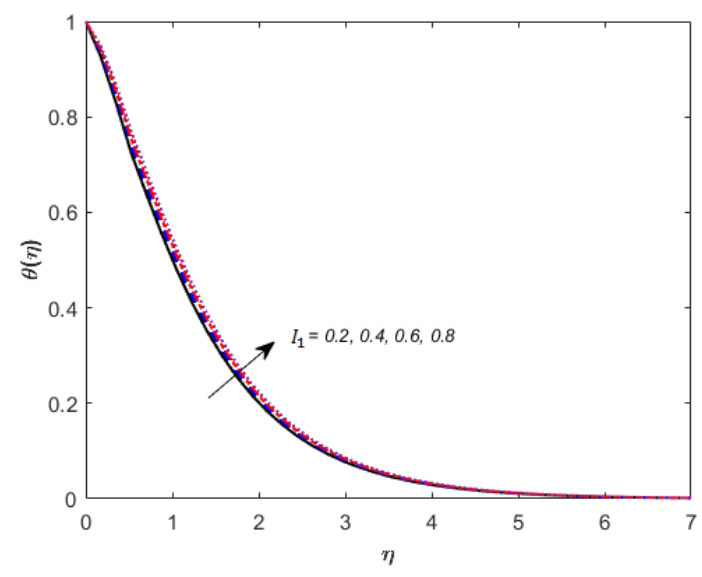

Fig. 20. Temperature $\theta$ verses $I_{1}$ when $\beta_{i}=$ $0.1, \alpha=0.1, \operatorname{Pr}=7, E c=0.2, n=1.1, \beta_{e}=$ $0.3, N_{R}=2.5, \theta_{w}=0.3, H a=0.8, I_{2}=0.4$ and $\varphi=0.05$. 


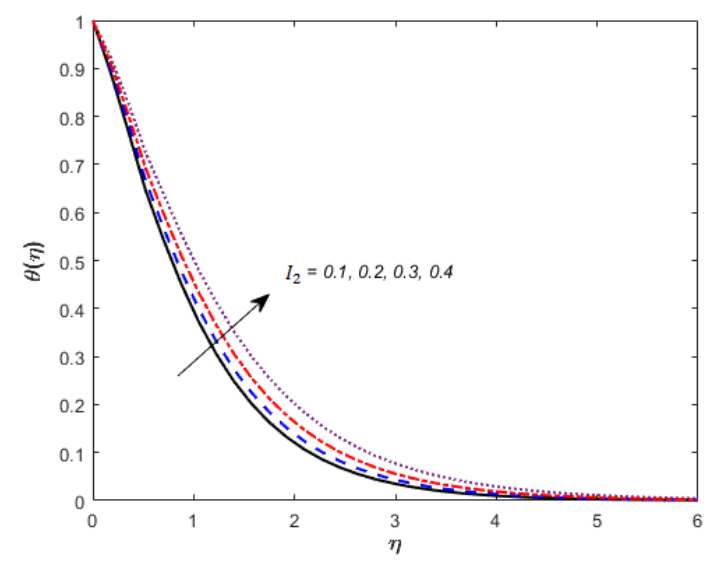

Fig. 21. Temperature $\theta$ verses $I_{2}$ when $\beta_{i}=$ $0.1, \alpha=0.1, \operatorname{Pr}=7, E c=0.2, n=1.1, \beta_{e}=$ $0.3, N_{R}=2.5, \theta_{w}=0.3, I_{1}=0.2, H a=0.8$ and $\varphi=0.05$.

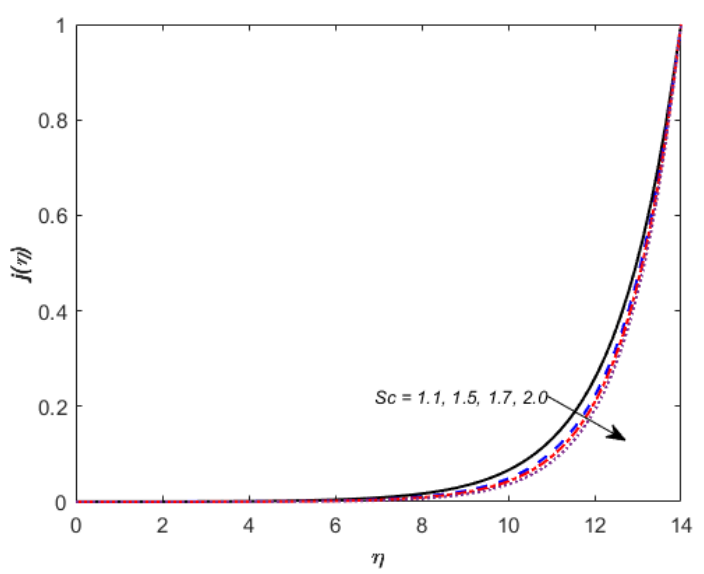

Fig. 23. Concentration $j$ verses $S c$ when $n=1.1, K^{*}=0.8$ and $H a=0.8$.

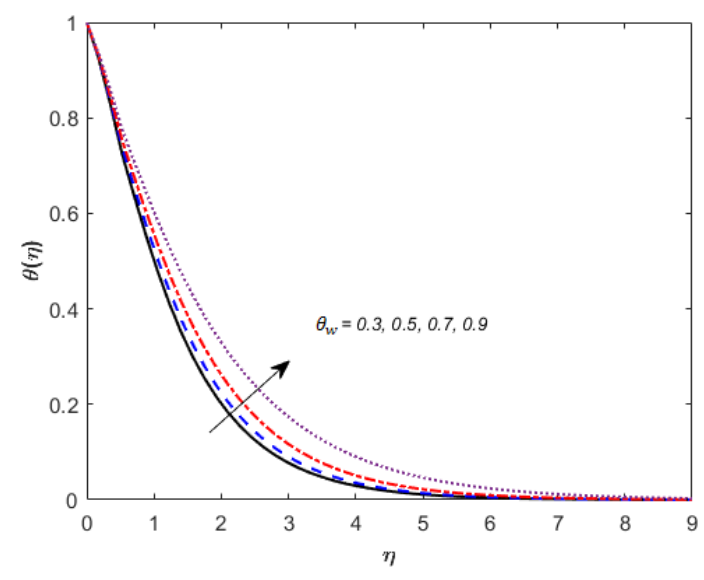

Fig. 22. Temperature $\theta$ verses $\theta_{w}$ when $\beta_{i}=0.1, \operatorname{Pr}=7, E c=0.2, n=1.1, \beta_{e}=$ $0.3, N_{R}=2.5, H a=0.8, I_{1}=0.2, I_{2}=0.4$ and $\varphi=0.05$.

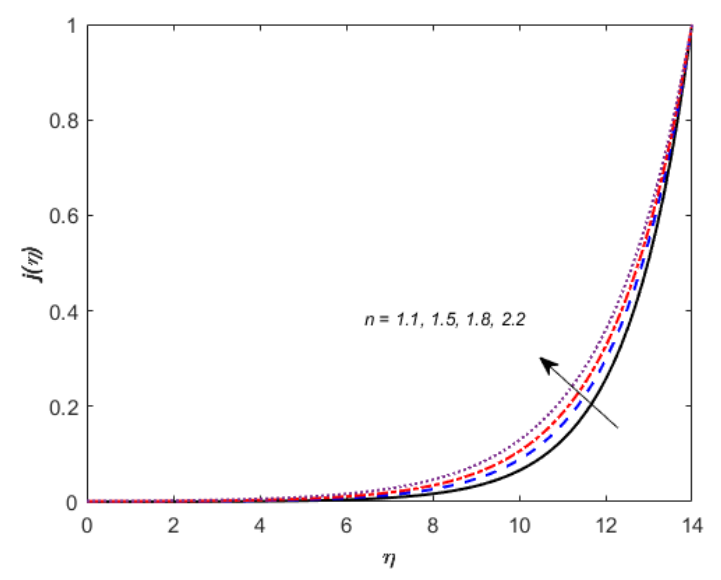

Fig. 24. Concentration $j$ verses $n$ when $\varphi=0.05, K^{*}=0.8$ and $S c=1.1$. 


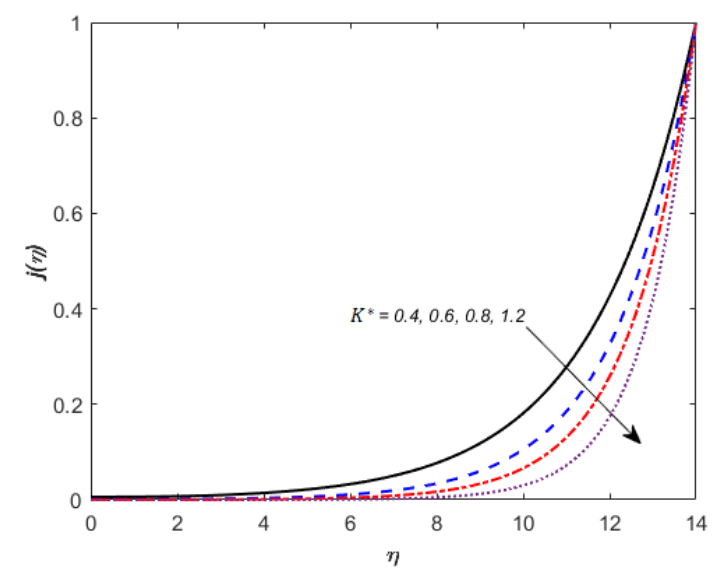

Fig. 25. Concentration $j$ verses $K^{*}$ when $\varphi=0.05, n=1.1$ and $S c=1.1$.

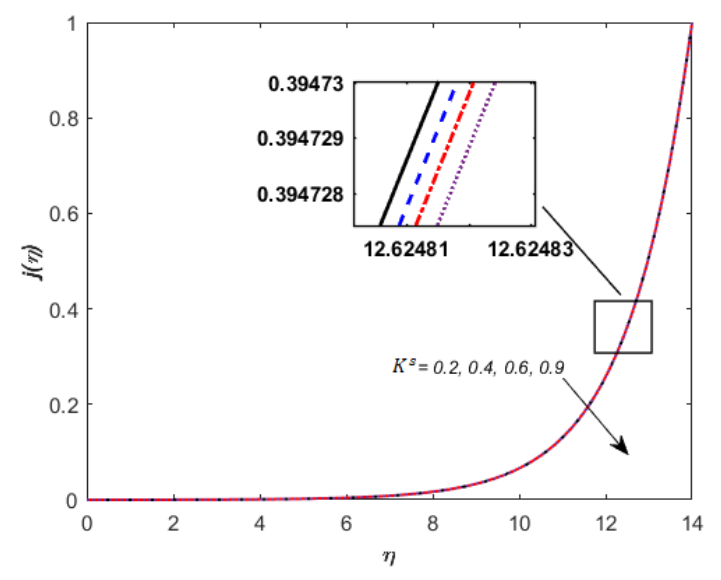

Fig. 26. Concentration $j$ verses $K^{s}$ when $n=1.1, K^{*}=0.8$ and $S c=1.1$.

Normalized wall shear stresses and normalized wall heat flux: Numerical values of normalized wall shear stresses and wall heat flux verses, temperature parameter $\left(\theta_{\omega}\right)$, Hall parameter $\left(\beta_{e}\right)$, ion-slip parameter $\left(\beta_{i}\right)$, Hartmann number $(H a)$ and various types of nanosized metallic particles are tabulated in Table 2. It can be observed from numerical values given Table 2 that wall shear stresses in $x$-direction decreases when Hall and ion slip parameters are increased. However, wall shear stress in $y$-direction increases when Hall parameter is increased whereas wall shear stress is decreased by increasing the ion slip parameter. Wall heat transfer rate is greatly affected by electron collisions and ion collisions. During numerical experiments, it is noted that the wall heat transfer rate increases when ion slip parameter is increased whereas wall heat transfer rate is increased by an increase in Hall parameter. Normalized wall shear stresses increase when the intensity of magnetic fluid is increased. However, monotonic increase in normalized wall heat flux is noted when intensity of applied magnetic fluid is increased.

Table 2. Behavior of skin friction, coupled stress tensor and Nusselt Number when $H a=0.8, \beta_{i}=0.1, \varphi=0.05, \alpha=0.1, \operatorname{Pr}=7, E c=0.2, n=1.1, K^{*}=0.8, \beta_{e}=0.3, N_{R}=$ $2.5, \theta_{w}=0.3, I_{1}=0.2, I_{2}=0.4$ and $S c=1.1$. 


\begin{tabular}{|c|c|c|c|c|}
\hline & & $-(\mathrm{Re})^{1 / 2} C_{f}$ & $-(\mathrm{Re})^{1 / 2} C_{g}$ & $(\mathrm{Re})^{-1 / 2} N u$ \\
\hline \multirow{4}{*}{$\theta_{w}$} & 0.3 & 2.543571632 & 0.212611636 & 0.244031364 \\
& 0.5 & 2.543571632 & 0.212611636 & 0.219946792 \\
& 0.7 & 2.543571632 & 0.212611636 & 0.004445535 \\
& 1.1 & 2.543571634 & 0.212611636 & 0.956173747 \\
\hline \multirow{4}{*}{$\beta_{e}$} & 0.9 & 2.530138242 & 0.246096562 & 0.329511893 \\
& 1.4 & 2.501604648 & 0.234687072 & 0.366162336 \\
& 1.7 & 2.491380161 & 0.224252695 & 0.384332910 \\
& 2.2 & 2.480883949 & 0.207556755 & 0.407318673 \\
\hline \multirow{4}{*}{$\beta_{i}$} & 0.1 & 2.587480168 & 0.211797480 & 0.298985296 \\
& 0.3 & 2.582126962 & 0.204451691 & 0.312903394 \\
& 0.9 & 2.568345436 & 0.187109225 & 0.345309682 \\
& 1.2 & 2.562527995 & 0.180396652 & 0.357683499 \\
\hline \multirow{5}{*}{$\varphi$} & 0.05 & 2.543571632 & 0.212611636 & 0.244031364 \\
& 0.15 & 3.380099096 & 0.281423911 & 0.541027309 \\
& 0.2 & 3.892053589 & 0.324070065 & 0.397616088 \\
& 0.3 & 5.199749524 & 0.433817620 & 0.339205752 \\
\hline \multirow{5}{*}{$H a$} & 0.3 & 2.465687743 & 0.151270649 & 0.158153720 \\
& 0.5 & 2.496761427 & 0.177513539 & -0.069963897 \\
& 0.7 & 2.527958808 & 0.201359739 & -0.306751703 \\
& 0.9 & 2.559182906 & 0.223490899 & -0.569979651 \\
\hline
\end{tabular}

\section{Conclusion}

Simultaneous effects of dispersion of nanosized particles, Hall and ion-slip currents thermal radiation and Joule heating in partially ionized liquid in the presence of chemically reacting species are investigated numerically. Extensive numerical experiments are conducted to note the behavior of flow fields. The key observations are listed below.

- The flow of magnetized nano-liquid speeds up as the collision rates of electrons and ions are increased. Electron collisions and ion collisions cause currents called Hall and ion slip currents respectively. These currents make the moving fluid to experience forces which are opposite to the magnetic force (an opposing force) in this case. So 
it is concluded that the Hall and ion forces reduce the impact of magnetic force in $x$-direction. Consequently, flow of nano-liquid in $x$-direction speeds up. Hence, our theoretical/mathematical analysis is completely in line with underlying physics. However, the impact of Hall force on fluid in $y$-direction is opposite to the impact of force due to ion slip current on fluid. Moreover, motion of nano-liquid slows down as the intensity of the magnetic field is increased.

- The influence of heterogeneous and homogeneous reaction on the concentration of reacting species is analyzed. It is observed that influence of homogeneous reaction on the concentration of species is more significant as compare to the effect of heterogeneous reaction. It is also noted that concentration of reacting species decreases when homogeneous and heterogeneous reaction rates are increased.

- The temperature of nano-liquid in the presence of magnetic field increases when the intensity of the magnetic field is increased. This increase in the temperature of the fluid is due to Ohmic and viscous dissipations. However, the collisions of electrons and collisions of ions cause a significant decrease in the temperature of partially ionized fluid. Thermal radiations play a vital role in the temperature of nano-liquid. It is observed that the temperature of nano-liquid decreases if it emits thermal radiations. A notable decline in thermal boundary layer thickness is observed.

- Effective thermal conductivity plays a significant role in the enhancement of heat transfer in liquids. Conducted theoretical analysis has revealed that $\mathrm{Fe}_{3} \mathrm{O}_{4}$ nano-particles give highest effective thermal conductivity. Therefore, dispersion of $\mathrm{Fe}_{3} \mathrm{O}_{4}$ particles is responsible of highest enhancement of heat transfer as compare to $\mathrm{Ag}, \mathrm{Al}_{2} \mathrm{O}_{3}$ and $\mathrm{TiO}_{2}$ nano-particles.

- The temperature of partially ionized liquid rises due to nonlinear non-uniform heat generation. The power-law index and heat generation have opposite effect on temperature and thermal boundary layer thickness.

Conflict of interest: There are no conflicts to declare

Acknowledgement: Fisrt two authors are thankful to the Higher Education Commission (HEC) of Pakistan for the financial support under NRPU-vide 5855/Federal/NRPU/R\&D/HEC/2016. 


\section{References}

[1] J. H. Merkin, A model for isothermal homogenous-heterogeneous reactions in boundarylayer flow. Math. Computer Model, 1994,.24, 125-136.

[2] T. Hayat, M. Rashid and A. Alsaedi, Three dimensional radiative flow of magnetitenanofluid with homogeneous-heterogeneous reactions, Results in Phy., 2018, 8, 268-275.

[3] N. Bachok, A. Ishak and I. Pop, On the stagnation-point flow towards a stretching sheet with homogeneous-heterogeneous reactions effects, Commun. Nonlinear Sci. Numer. Simul., 2011, 16, 4296-302.

[4] B. J. Gireesha, P. B. S. Kumar, B. Mahanthesh, S. A. Shehzad and A. Rauf, Nonlinear 3D flow of Casson-Carreau fluids with homogeneous-heterogeneous reactions: a comparative study, Res. Phys., 2017, 7, 2762-70.

[5] T. Hayat, M. Rashid, M. Imtiaz and A. Alsaedi, Nanofluid flow due to rotating disk with variable thickness and homogeneous-heterogeneous reactions, Int. J. Heat Mass Transfer, 2017, 113, 96-105.

[6] M. Sajid, S. A. Iqbal, M. Naveed and Z. Abbas, Effect of homogeneous-heterogeneous reactions and magnetohydrodynamics on $\mathrm{Fe}_{3} \mathrm{O}_{4}$ nanofluid for the Blasius flow with thermal radiations, J. Mol. Liq., 2017, 115-21.

[7] S. Qayyum, M. I. Khan, T. Hayat and A. Alsaedi, A framework for nonlinear thermal radiation and homogeneous-heterogeneous reactions flow based on silverwater and copper-water nanoparticles: A numerical model for probable error, Res. Phys., 2017, 7, $1907-14$.

[8] M. Nawaz, S. Rana, I. H. Qureshi, and T. Hayat, Three-dimensional heat transfer in the mixture of nanoparticles and micropolar MHD plasma with Hall and ion slip effects, AIP Advances, 2018, 8, 105-109.

[9] Y. V. Nazarov, Generalized Ohm's Law, Quantum Dynamics of Submicron Structures, 1995, 687-704.

[10] T. Hayat and M. Nawaz, hall and ion-slip effects on three-dimensional flow of a second grade fluid, Int. J. for Num. Methods in Fluids, 2011, 66(2), 183-193. 
[11] S. Motsa and S. Shateyi, The effects of chemical reaction, Hall and ion-slip currents on MHD micropolar fluid flow with thermal diffusivity using a novel numerical technical, J. Appl. Math., 2012, 30.

[12] S. Das, R. Jana and O. Makinde, Magnetohydrodynamic free convective flow of nanofluid past an oscillating porous flat plate in a rotating system with thermal radiation and Hall effects, J. Mech., 2016, 32(2), 197-210.

[13] M. Nawaz and T. Zubair, Finite element study of three dimensional radiative nanoplasma flow subject to Hall and ion slip currents, Results in Physics, 2017, 7, 4111-4122.

[14] T. Hayat, H. Zahir, A. Alsaedi and B. Ahmad, Hall current and Joule heating effects on peristaltic flow of viscous fluid in a rotating channel with convected boundary conditions, Res. Phys., 2017, 7, 2831-2836.

[15] S. I. Abdelsalam and M. M. Bhatti, The study of non-Newtonian nanofluid with Hall and ion-slip effects on peristaltically induced motion in a non-uniform channel, RSC Adv., 2018, 8(15), 7904-7915.

[16] E. M. Abo-Eldahab and M. A. El Aziz, Viscous dissipation and Joule heating effects on MHD free-convection from a vertical plate with power-law variation in surface temperature in the presence of Hall and ion-slip currents, Appl. Math. Modelling, 2005, 29(6), $579-595$.

[17] M. Nawaz, S. Rana and I. H. Qureshi, Computational fluid dynamic simulations for dispersion of nanoparticles in a magnetohydrodynamic liquid: a Galerkin finite element method, RSC Adv., 2018, 8, 38324-38335.

[18] O. Odelu and N. N. Kumar, Slip flow and heat transfer of chemically reacting micropolar fluid through expanding or contracting walls with Hall and ion-slip currents, Ain Shams Eng. J., 2018, 9(1), 137-147.

[19] B. N. M. da Silva, G. E. Assad and J. A. de Lima, Unsteady Magnetohydrodynamic channel flow with Hall and ion-slip effects: The integral transform solution procedure, J. of Enhanced Heat Transfer, 2018, 25(4-5), 367-386. 
[20] J. Reza, F. Mebarek-Oudina and O. D. Makinde, MHD Slip flow of $C u$-Kerosene Nanofluid in a channel with stretching walls using 3-stage lobatto IIIA formula, Defect and Diffusion Forum, 2018, 387, 51-62.

[21] M. Nawaz, S. Rana, I. H. Qureshi, and T. Hayat, Three-dimensional heat transfer in the mixture of nanoparticles and micropolar MHD plasma with Hall and ion slip effects, AIP Advances, 2018, 8, 105-109.

[22] I. H. Qureshi, M. Nawaz, S. Rana, and T. Zubair, Galerkin Finite Element Study on the Effects of Variable Thermal Conductivity and Variable Mass Diffusion Conductance on Heat and Mass Transfer, Commun. Theor. Phys., 2018, 70(1), 49-59.

[23] I. H. Qureshi, M. Nawaz, S. Rana, U. Nazir, and A. J. Chamkha, Investigation of variable thermo-physical properties of viscoelastic rheology: A Galerkin finite element approach, AIP Advances, 2018, 8, 075027. 